\title{
Research Paper \\ The Relationship Between Underlying Factors and Treatment Adherence in Women With Gestational Diabetes
}

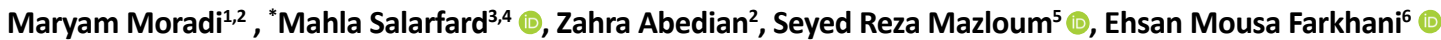

1. Nursing and Midwifery Care Research Center, Mashhad University of Medical Sciences, Mashhad, Iran.

2. Department of Midwifery, School of Nursing and Midwifery, Mashhad University of Medical Sciences, Mashhad, Iran.

3. Student Research Committee, School of Nursing and Midwifery, Mashhad University of Medical Sciences, Mashhad, Iran.

4. Department of Midwifery, School of Nursing and Midwifery, Birjand University of Medical Sciences, Birjand, Iran.

5. Department of Nursing, School of Nursing and Midwifery, Mashhad University of Medical Sciences, Mashhad, Iran.

6. Department of Epidemiology, School of Health, Mashhad University of Medical Sciences, Mashhad, Iran.

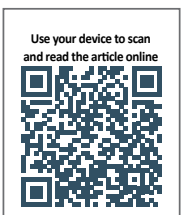

Citation: Moradi M, Salarfard M, Abedian Z, Mazloum SR, Mousa Farkhani E. [The Relationship Between Underlying Factors and Treatment Adherence in Women With Gestational Diabetes (Persian)]. Journal of Arak University of Medical Sciences(JAMS). 2021; 23(6):828-839. https://doi.org/10.32598/JAMS.23.6.6192.1

Key words:

Pregnancy, Treatment Adherence, Gestational diabetes

\section{A B STRACT}

Article Info:

Received: 20 May 2020

Accepted: 12 Aug 2020

Available Online: 01 Feb 2021

Background and Aim The adherence of diabetic patients to the recommended treatment regimens is among the major challenges in diabetes control. Dietary adherence is low in pregnant women with gestational diabetes. Non-compliance can reduce the beneficial effects of treatment, symptoms, and signs, increase complications, or even cause death. Therefore, further research on the determinants of followup behaviors is essential.

Methods \& Materials This was a descriptive-analytical study. In total, 260 women with gestational diabetes treated with diet referring to healthcare centers, and Imam Reza (AS), Ghaem, and Umm Al-Banin Hospitals in Mashhad City, Iran participated in this research. The study subjects were selected using convenience, multistage, class, and cluster sampling methods. The required data were collected using a demographic and pregnancy profile questionnaire and a researcher-made questionnaire of adherence to the treatment regimen. The obtained data were analyzed by inferential statists and regression tests in SPSS v. 16.

Ethical Considerations This study was registered with ethics code of IR.MUMS.NURSE.REC.1397.091 in the ethics committee of Mashhad University of Medical Sciences.

Results The regression analysis results indicated that dietary adherence, blood glucose monitoring, and exercise adherence scores presented a significant and positive effect on the total treatment adherence score $(P<0.001)$. Furthermore, education $(P=0.044)$, unwanted $(P=0.006)$, or unplanned pregnancy $(P=0.21)$ provided a positive and significant effect on the overall score of treatment adherence. Women with a history of diabetes in first-degree relatives $(P=0.010)$ and those with a history of miscarriage $(P=0.021)$ obtained higher scores of total treatment adherence.

Conclusion According to the present study findings, some demographic and pregnancy characteristics can affect the rate of adherence to the treatment regimen in women with gestational diabetes. Healthcare providers need to pay attention to the impact of these factors in improving adherence to patients' treatment regimens.

\section{"Corresponding Author:}




\section{Extended Abstract}

\section{Introduction}

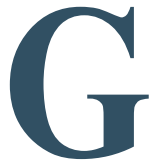

estational diabetes is the most prevalent metabolic disorder in pregnancy [1]. The adherence of diabetic patients to the recommended treatment regimens is among the major challenges in diabetes control

[6]. The acceptance of physicians' recommendations, including medication, diet, and exercise, and adherence to it leads to the control of the patient's blood glucose; it also plays a decisive role in predicting complications associated with diabetes [8]. Studies reported that following medication and care instructions (diet, blood glucose monitoring, $\&$ exercise) is insufficient [9]. Failure to follow can reduce the beneficial therapeutic effects, signs, and symptoms, increase complications, or even cause death. Therefore, further research on the determinants of adherence to treatment regimens seems necessary [11]. Previous studies revealed that some individual and social characteristics can affect the rate of adherence to the treatment regimen [13]. Therefore, the present study aimed to determine the relationship between demographic characteristics and pregnancy, and adherence to treatment in patients with gestational diabetes.

\section{Materials and Methods}

This descriptive-analytical study was conducted in 2019. The study subjects were 260 women with gestational diabetes treated with diet referring to healthcare centers and hospitals of Imam Reza, Ghaem, Umm Al-Banin. The study participants were selected by multistage integration sampling method in Mashhad City, Iran. The necessary data were collected using a demographic and pregnancy information checklist and a researcher-made questionnaire on adherence to treatment. The obtained demographic and pregnancy information included age; education; occupation; monthly income; gestational diabetes information; history of diabetes in first-degree relatives; history of gestational diabetes; the number of pregnancies; history of miscarriage; stillbirth and infertility, and whether or not the pregnancy was desired. To measure adherence to diet, exercise, and blood glucose monitoring, a researcher-made questionnaire was used; it includes 17 questions answered on a Likert-type scale. Moreover, 12 questions are related to diet, 2 questions concern exercise and 3 questions address blood glucose monitoring (it included two parts of self-monitoring \& venous blood glucose monitoring). The validity of the tools was determined using the Content Validity Index (CVI). The validity of the researcher-made adherence to treatment questionnaire was measured by a CVI of 0.97 and a Content Validity Ratio (CVR) of 0.89 .
The reliability of the questionnaire of diet adherence, blood glucose monitoring, and exercise was determined using Cronbach's alpha coefficient of 0.83 . Data analysis was performed in SPSS by the statistical indices of mean, standard deviation, frequency distribution, and multiple linear regression.

\section{Results}

The frequency distribution of women with gestational diabetes respecting demographic and fertility characteristics is presented in Table 1. The scores of adherence to the treatment regimen and its components in women with gestational diabetes are illustrated in Table 2.

The regression analysis results of all demographic and pregnancy variables with the total score of treatment adherence reflected that diet adherence, blood glucose monitoring, and exercise adherence scores provided a significant and positive correlation with total treatment adherence score $(\mathrm{P}<0.001)$. According to the standardized coefficient values, diet compliance, blood glucose monitoring, and exercise adherence scores presented the greatest correlation with the total treatment adherence score, respectively. The obtained results also signified that individuals with university education had a higher total score of treatment adherence, compared to the subjects with reading and writing education ( $\mathrm{P}=0.044)$. Unwanted $(\mathrm{P}=0.006)$ or unplanned $(\mathrm{P}=0.021)$ pregnancies were positively and significantly correlated with the total score of treatment adherence. Women with a history of first-degree diabetes $(\mathrm{P}=0.010)$ and women with a history of abortion $(\mathrm{P}=0.021)$ achieved higher scores of total treatment adherence. In other variables, there was no significant correlation with the total score of treatment adherence (Table 3).

\section{Discussion and Conclusion}

The present study results revealed that adherence to diet score, blood glucose monitoring score, and adherence to exercise score presented a significant and positive relationship with the total treatment adherence score. Hernández-Ronquillo et al. (2003) also documented that patients who followed the instructions related to physical activity and exercise were successful in controlling diabetes [17]. Furthermore, the obtained data signified a positive and significant correlation between the level of education and the total score of adherence to the treatment regimen. According to Abedi et al. (2015), there was a significant and positive association between following the treatment regimen of type 2 diabetes and education [16]. In the present study, no significant relationship was found between the extent of information (according to the subject) about gestational 
Table 1. Frequency distribution of women with gestational diabetes according to demographic and pregnancy characteristics (260 people)

\begin{tabular}{|c|c|c|c|}
\hline Variable & No. (\%) & Variable & No. (\%) \\
\hline \multicolumn{2}{|c|}{ Age, $y$} & \multicolumn{2}{|c|}{ Education level } \\
\hline $17-24$ & $26(10.0)$ & Reading and writing & $14(5.4)$ \\
\hline $25-29$ & $52(20.0)$ & Primary school & $61(23.5)$ \\
\hline $30-34$ & $75(28.8)$ & Secondary and high school & $150(57.7)$ \\
\hline $35-48$ & $107(41.2)$ & Academic & $35(13.5)$ \\
\hline \multicolumn{2}{|c|}{ Monthly income } & \multicolumn{2}{|c|}{ Gestational diabetes information } \\
\hline Less than enough & $96(36.9)$ & Low & $177(68.1)$ \\
\hline Enough & $159(61.2)$ & Moderate & $81(31.2)$ \\
\hline More than enough & $5(1.9)$ & High & $2(0.8)$ \\
\hline \multicolumn{2}{|c|}{ Occupation } & \multicolumn{2}{|c|}{ History of abortion } \\
\hline Housewife & $244(93.8)$ & No & $177(68.1)$ \\
\hline Employed & $16(6.2)$ & Yes & $83(31.9)$ \\
\hline \multicolumn{2}{|c|}{ History of diabetes in first-degree relatives } & \multicolumn{2}{|c|}{ History of stillbirth } \\
\hline No & $148(56.9)$ & No & $245(94.2)$ \\
\hline Yes & $112(43.1)$ & Yes & $15(5.8)$ \\
\hline \multicolumn{4}{|c|}{ History of gestational diabetes } \\
\hline \multicolumn{2}{|c|}{ No } & \multicolumn{2}{|c|}{$190(73.1)$} \\
\hline \multicolumn{2}{|c|}{ Yes } & \multicolumn{2}{|c|}{70 (26.9) } \\
\hline \multicolumn{2}{|c|}{ Number of pregnancies } & \multicolumn{2}{|c|}{ History of infertility } \\
\hline 1 & $44(16.9)$ & No & $232(89.2)$ \\
\hline 2 & $70(26.9)$ & Yes & $28(10.8)$ \\
\hline 3 & $58(22.3)$ & Wanted or u & lancy \\
\hline \multirow{3}{*}{$\geq 3$} & & Wanted & $213(81.9)$ \\
\hline & & Unwanted & $35(13.5)$ \\
\hline & & Unplanned & $12(4.6)$ \\
\hline
\end{tabular}

Table 2. The scores of adherence to treatment and its components in women with gestational diabetes $(n=260)$

\begin{tabular}{cc}
\hline Variable & Mean \pm SD \\
\hline Adherence to diet & $24.3 \pm 3.7$ \\
\hline Blood glucose monitoring & $4.6 \pm 2.4$ \\
Adherence to exercise & $1.6 \pm 1.5$ \\
\hline The total score of adherence to treatment & $30.5 \pm 5.7$ \\
\hline
\end{tabular}


Table 3. Results of Multiple Linear Regression Analysis of the Relationship between Demographic Variables and Components of Treatment Adherence to Total Adherence Score

\begin{tabular}{|c|c|c|c|c|c|c|}
\hline \multicolumn{2}{|c|}{ Variable } & Coefficient & $\begin{array}{l}\text { Standard Coef- } \\
\text { ficient Deviation }\end{array}$ & $\begin{array}{c}\text { Standardized } \\
\text { Coefficient }\end{array}$ & $\mathbf{t}$ & $\mathbf{p}$ \\
\hline \multicolumn{2}{|c|}{ Constant } & 1.039 & 0.406 & & 2.558 & 0.011 \\
\hline \multicolumn{2}{|c|}{ Diet adherence score } & 0.982 & 0.012 & 0.643 & 80.297 & $<0.001$ \\
\hline \multicolumn{2}{|c|}{ Blood glucose monitoring score } & 0.960 & 0.016 & 0.409 & 60.656 & $<0.001$ \\
\hline \multicolumn{2}{|c|}{ Sports adherence score } & 0.964 & 0.027 & 0.251 & 35.173 & $<0.001$ \\
\hline \multicolumn{2}{|l|}{ Age } & -0.003 & 0.006 & -0.003 & -0.460 & 0.646 \\
\hline Job (Reference: Housewife) & Employed & -0.210 & 0.164 & -0.009 & -1.282 & 0.201 \\
\hline \multirow{3}{*}{$\begin{array}{c}\text { Education (Reference: Reading } \\
\text { and Writing) }\end{array}$} & Elementary & 0.063 & 0.166 & 0.005 & 0.378 & 0.705 \\
\hline & Middle and high school & 0.117 & 0.167 & 0.010 & 0.697 & 0.486 \\
\hline & University & 0.430 & 0.212 & 0.026 & 2.022 & 0.044 \\
\hline \multirow{2}{*}{$\begin{array}{c}\text { Monthly income (reference: Less } \\
\text { than enough) }\end{array}$} & Enough & -0.028 & 0.079 & -0.002 & -0.356 & 0.722 \\
\hline & More than enough & -0.372 & 0.280 & -0.009 & -1.327 & 0.186 \\
\hline \multirow{2}{*}{$\begin{array}{l}\text { Pregnancy diabetes information } \\
\text { (Reference: Low) }\end{array}$} & Medium & 0.141 & 0.081 & 0.012 & 1.744 & 0.082 \\
\hline & Much & 0.332 & 0.404 & 0.005 & 0.823 & 0.411 \\
\hline \multirow{2}{*}{$\begin{array}{c}\text { Pregnancy request (Reference: } \\
\text { Request) }\end{array}$} & Unintentionally & 0.296 & 0.107 & 0.018 & 2.754 & 0.006 \\
\hline & Unplanned & 0.387 & 0.167 & 0.014 & 2.323 & 0.021 \\
\hline \multicolumn{2}{|c|}{ Gravida } & 0.021 & 0.030 & 0.003 & 0.400 & 0.689 \\
\hline \multicolumn{2}{|c|}{ History of diabetes in first-degree relatives (reference: No) } & 0.190 & 0.073 & 0.017 & 2.597 & 0.010 \\
\hline \multicolumn{2}{|c|}{ History of gestational diabetes (reference: No) } & -0.071 & 0.084 & -0.006 & -0.843 & 0.400 \\
\hline \multicolumn{2}{|c|}{ Abortion history (reference: No) } & -0.196 & 0.085 & -0.016 & -2.324 & 0.021 \\
\hline \multicolumn{2}{|c|}{ History of stillbirth (reference: No) } & -0.027 & 0.153 & -0.001 & -0.177 & 0.859 \\
\hline \multicolumn{2}{|c|}{ Infertility history (reference: No) } & 0.003 & 0.113 & 0.001 & 0.030 & 0.976 \\
\hline
\end{tabular}

diabetes and the score of treatment adherence. In the study of Haji Arabi et al. (2018), no significant relationship was found between knowledge about diabetes and self-care in patients with gestational diabetes [20]. In the present study, unwanted or unplanned pregnancies were positively and significantly correlated with the total score of treatment adherence. Farahani Dastjani et al. (2015) observed an inverse relationship and a significant difference between the lack of motivation and adherence to pharmacotherapy [22]. In the present study, women with a history of miscarriage presented a higher adherence to treatment. Women with an abortion experience (an abortion caused by gestational dia- betes or any other reasons) and those who have lost their fetus seem to care more about their treatment regimen and manifest greater adherence to treatment.

The current research results suggested that some demographic and pregnancy characteristics were associated with adherence to treatment in women with gestational diabetes; thus, healthcare providers, especially midwives and gynecologists must improve adherence to treatment by considering the role of these factors in these patients. 


\section{Ethical Considerations}

\section{Compliance with ethical guidelines}

This study was approved by the Ethics Committee of Mashhad University of Medical Sciences (Code: IR.MUMS.NURSE.REC.1397.091).

\section{Funding}

This research was supported by the Vice-Chancellor for Research, School of Nursing and Midwifery, Mashhad University of Medical Sciences (Grant No.: 971109).

\section{Authors' contributions}

Conceptualization: Maryam Moradi, Zahra Abedian; Research and sampling method: Mahla Salarfard, Ehsan Musa Farkhani; Data analysis: Mahla Salarfard, Seyed Reza Mazloum and Maryam Moradi; Text Writing and Review: All Authors.

\section{Conflicts of interest}

The authors declared no conflicts of interest.

\section{Acknowledgements}

We would like to thank the Student Research Committee of Mashhad University of Medical Sciences and the director and staff of Emma Reza, Ghaem, and Umm Al-Banin hospitals, and healthcare centers in Mashhad who contributed to this research. 


\section{بررسى ارثباط عوامل زمينهاى با تبعيت از درمان در زنان مبثلا به ديابت باردارى}

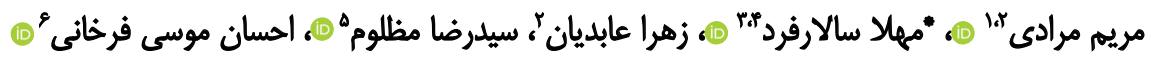

ا. مركز تحقيقات مراقبتهاى يرستارى و مامايى، دانشعاه علوميز شكى مشهيد، مشهيد، ايران.

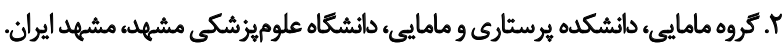

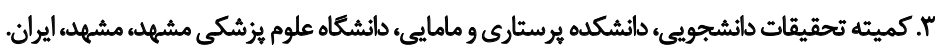

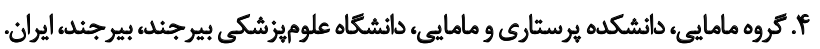

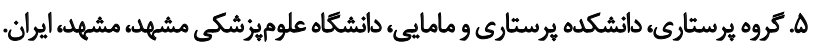

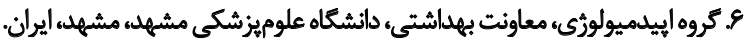

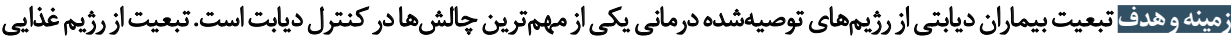

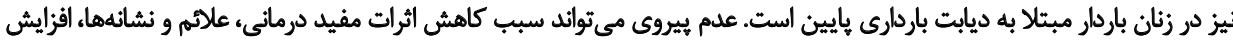

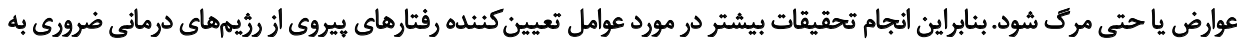

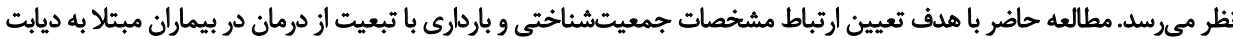
بارفارى انتجام شد.

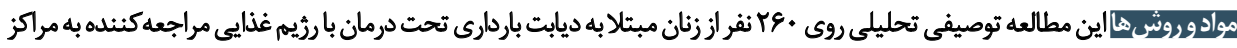

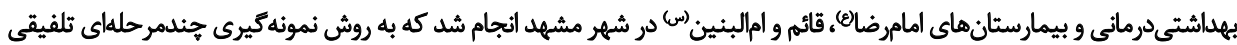

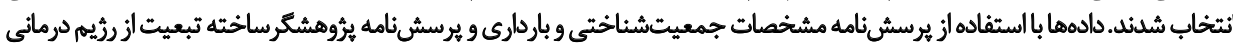

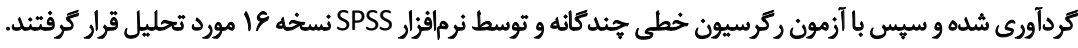

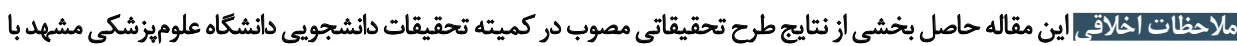

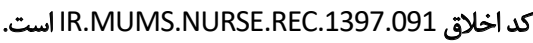

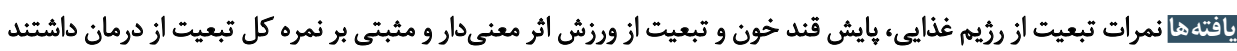

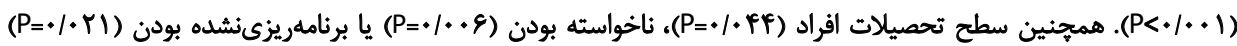

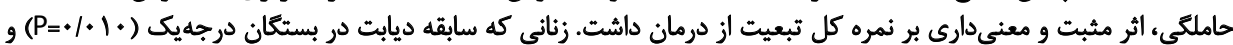

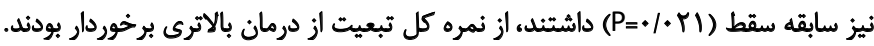

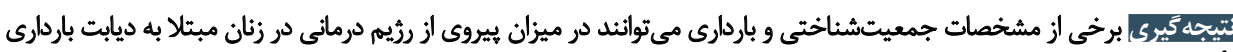

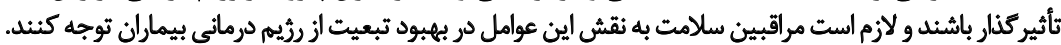

اطلاعات مقاله:

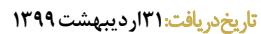
تاريخ يذيرش: Trار مرداد تاريخ انتشار: با بهمن

كليدوازوها:

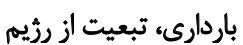
درمانى، ديابت باردارى تبعيت ازرئ

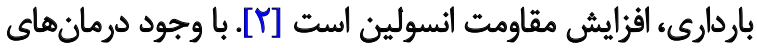

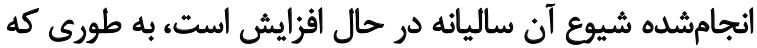

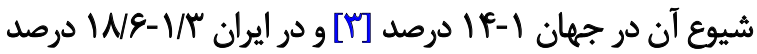

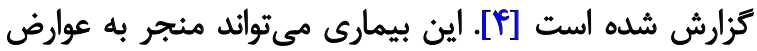

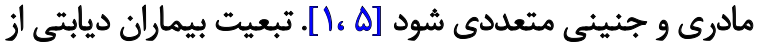

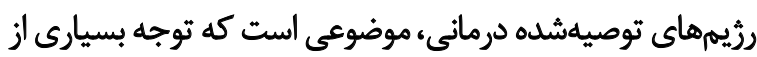

ديابت باردارى شايعترين اختلال متابوليك دوران باردارى بوده

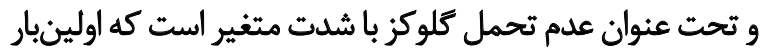

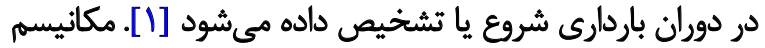
دقيق بروز ديابت باردارى مشخص نيست نيست. مشخصه اصلى دئى ديابت مكانيست 
مشاهده ميشود. يكى از عوامل مؤثر بر ميزان تبعيت بيماران،

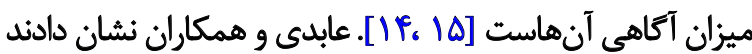

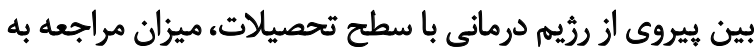

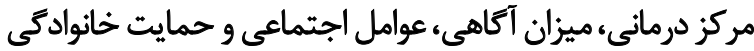

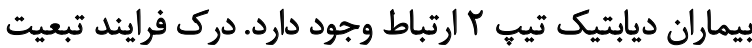

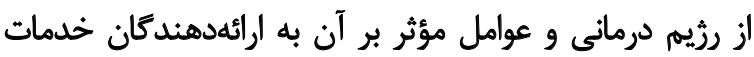

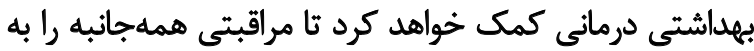

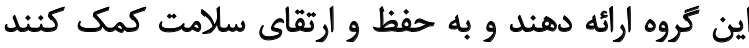

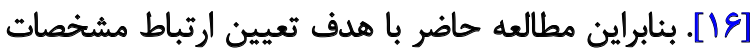

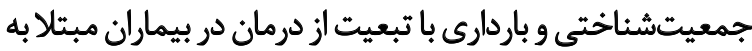

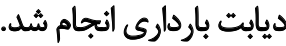

$$
\text { مواد و روشى باثنا }
$$

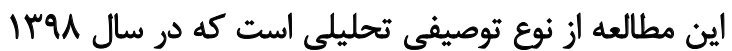

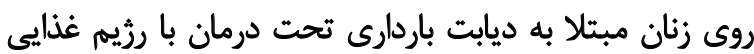

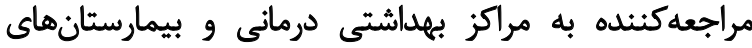

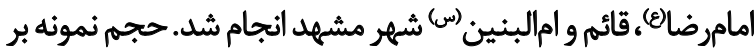

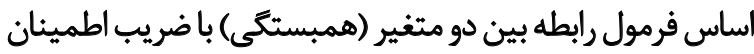

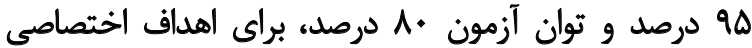

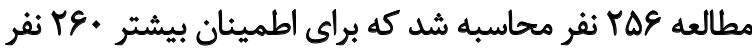

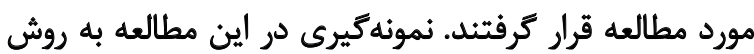

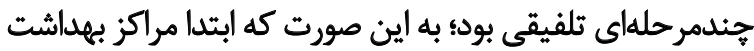
ينج كانه به عنوان طبقه در نظر كرئه

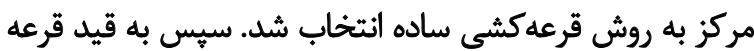

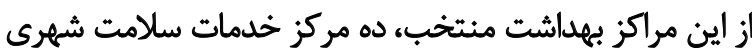

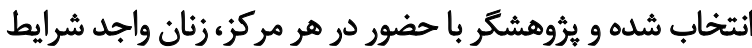

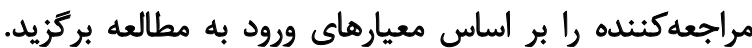

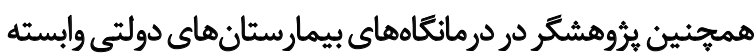

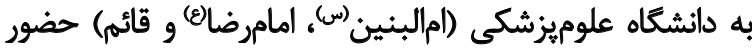

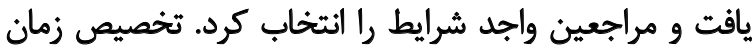

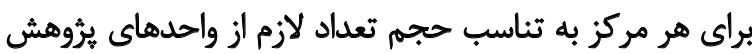

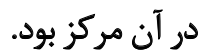

معيار هاى ورود به مطالعه عبارت بودند از: رضايت جهت شركت

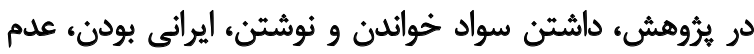

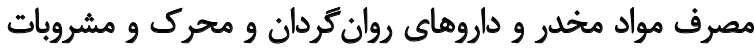

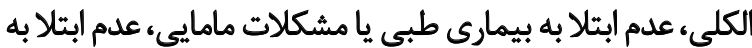

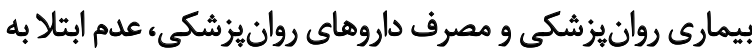

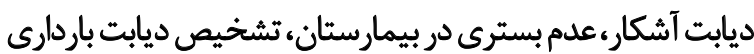

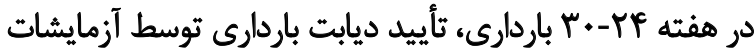

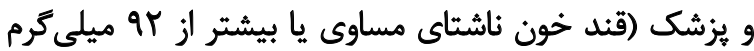

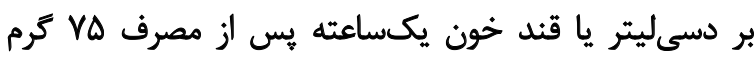

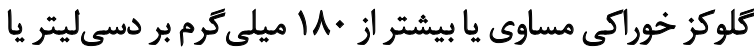

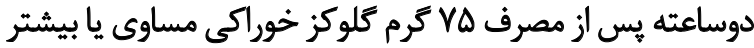

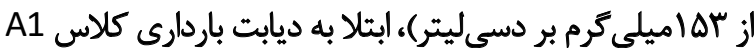

درمانكَران را به خود معطوف داشته و يكى از مههترين خالشها

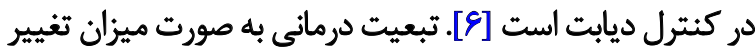

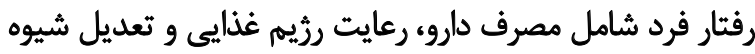

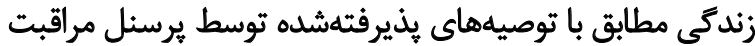

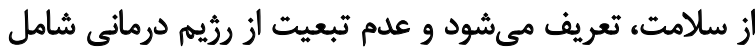

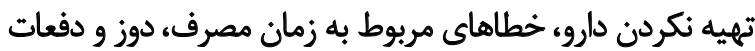

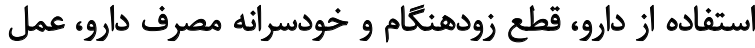

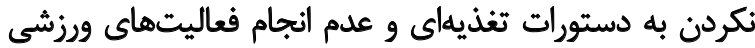

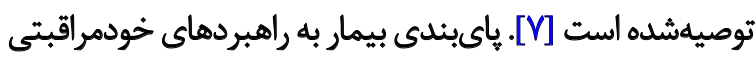

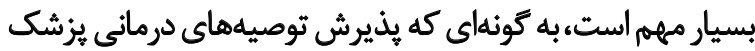

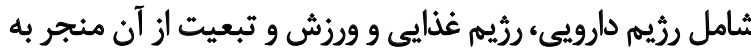

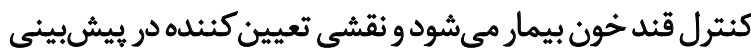
عوارض ناشى از ديابت دارد [1/].

على غم اهميت بيروى از دستورات مراقبتى، يافتههاى مطالعات

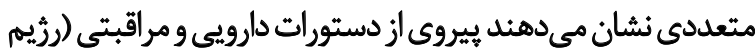

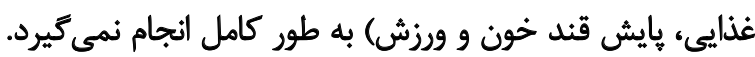

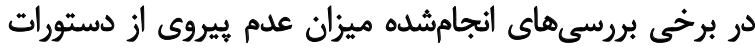

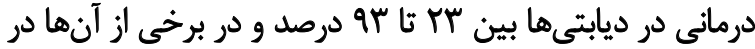

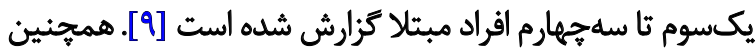

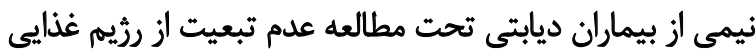

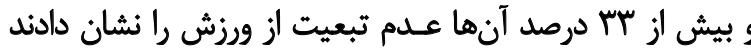

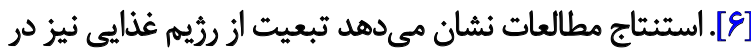

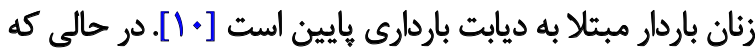

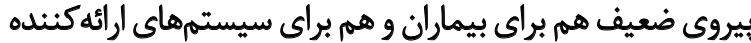

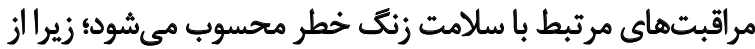

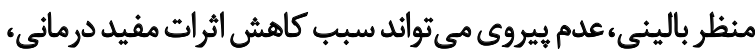

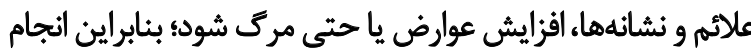

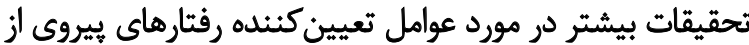

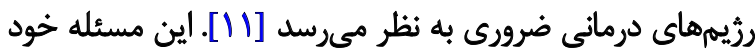

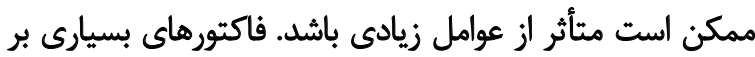

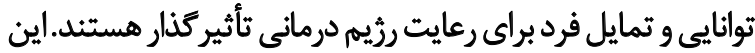

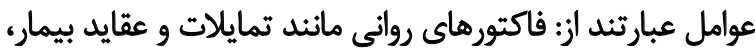

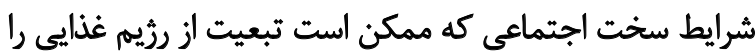

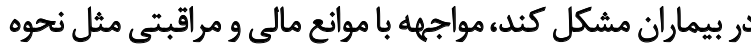

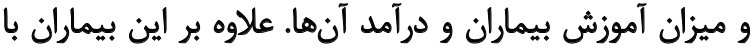

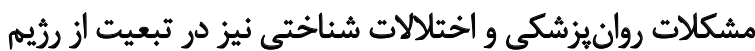

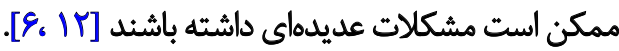

نتايج مطالعات نشان ميدهند برخى از عوامل فردى و وئرئ

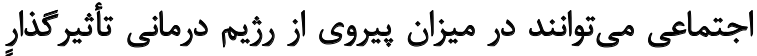

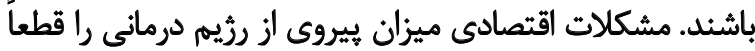

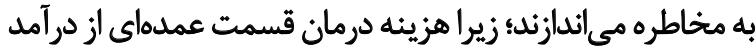

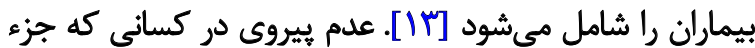

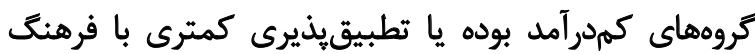

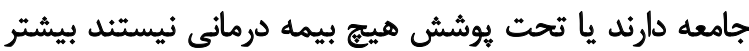


جدول ا. توزيع فراوائى زنان مبتلا به ديابت باردارى مورد مطالعه بر حسب خصوصيات جمعيتشناختى و باردارى (•\& نفر)

\begin{tabular}{|c|c|c|c|c|c|}
\hline تعداد (درصد) & \multicolumn{2}{|c|}{ متغيرها } & تعداد (درصد) & \multicolumn{2}{|c|}{ مثغيرها } \\
\hline If $\left(\Delta / \varphi^{\oplus}\right)$ & خواندن و نوشتن & & $\operatorname{re}(1.1 \%)$ & Mftr & \\
\hline$S)(\pi / \Delta)$ & ابتدايى & & $\operatorname{ar}(r+1+)$ & هrit & \\
\hline $1 \Delta \cdot(\Delta V / V)$ & راهنمايي و دييرستان & تحصيلات & $V \Delta(Y N A)$ & mér. & سن (سال) \\
\hline$r \Delta(M / \Delta)$ & دانشكاهى & & $1 \cdot V\left(f^{e} V / K\right)$ & PALto & \\
\hline $\operatorname{IW}($ ENI) & Sم & & $Q 8(r \& / q)$ & كمتر از حد كفاف & \\
\hline$\Lambda \backslash(M / T)$ & مثتوسط & اطاعات ديابت باردارى & $109(8 / / T)$ & در حد كفاف & درآمل ماهيانه \\
\hline$r(\cdot / \Lambda)$ & زياد & & $\Delta(1 / 9)$ & بيشتر از حلد كفاف & \\
\hline $\operatorname{IW}(\& N)$ & خير & 10 & $\operatorname{maf}(9 \Psi / \Lambda)$ & خائهدار & $1: 4$ \\
\hline$\lambda \mu(r / / q)$ & 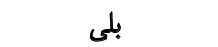 & & $18(\varepsilon / \pi)$ & شاغل & \\
\hline TYA $(9 F / T)$ & خير & & $\operatorname{lfA}(\Delta S / 9)$ & 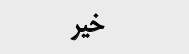 & صابقه ديابت در بستكان \\
\hline $10(\Delta / \Lambda)$ & بلى ا & البابه مردمرايلى & $\| r(r+1)$ & 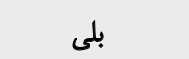 & درجهيك \\
\hline$M m(19 / r)$ & خير & & $19 \cdot(n+1)$ & خير & \\
\hline $\operatorname{rA}(1 \cdot / A)$ & بليى & سابقه نازايي & $V \cdot(r g / q)$ & 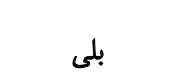 & سابقه ديابت باردارى \\
\hline $\operatorname{rir}(A / / 9)$ & خواسته & & $\operatorname{Pf}(18 / 9)$ & يكبار & \\
\hline$r \Delta(1 Y / \Delta)$ & ناخواسته & خواسته يا ناخواسته بودن & $V \cdot(Y \& / 9)$ & دوبار & تعداد باردارى \\
\hline $\operatorname{Ir}(s / 9)$ & برنامهريزىنشلده & & Iff $(\Delta F / 1)$ & سلبار و بيشتر & \\
\hline
\end{tabular}

به صورت مقياس ليكرت (هركز، بهندرت، اغلب اوقات و هميشه)

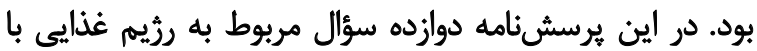

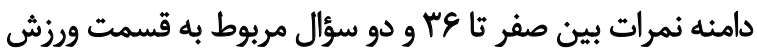

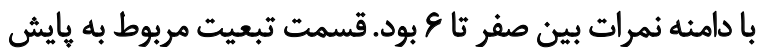

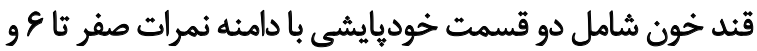

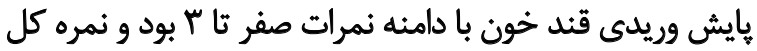

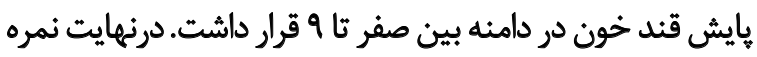

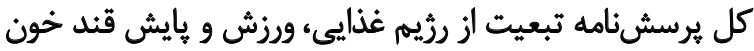

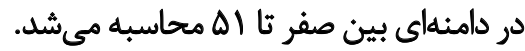

روايى ابزارها با استفاده از روايى محتوا تعيين شد؛ بلدين صورت

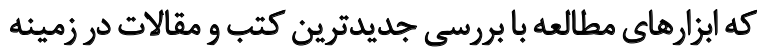

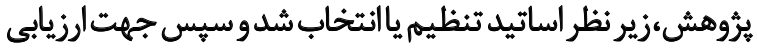

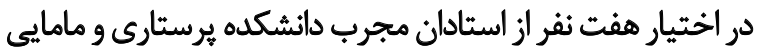

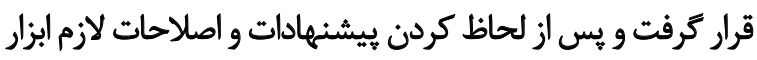

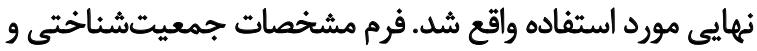

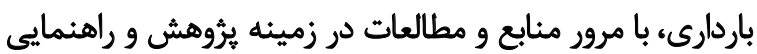

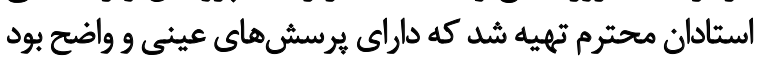

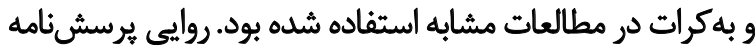

با توجه به نتيجه آزمايش قند خون ناشتاو دو ساعت بعد از غذا

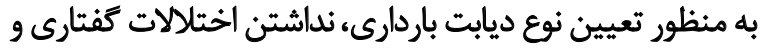

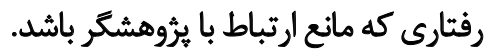

يس از توضيح هدف مطالعه به واحدهاي يثروهش واجد

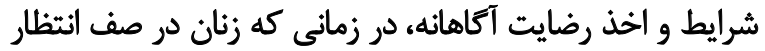

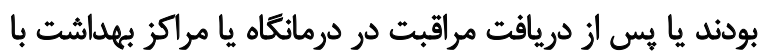

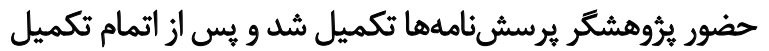

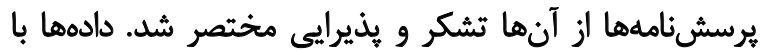

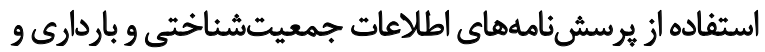

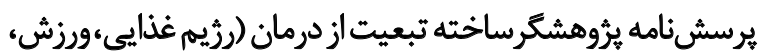

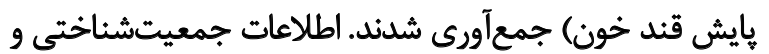

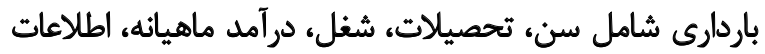

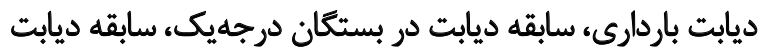

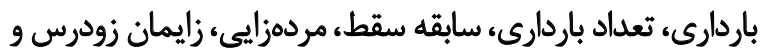

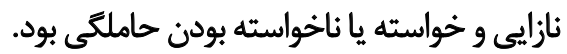

جهت سنجش تبعيث از رثيم غذايى، ورزش و يايش قند خون

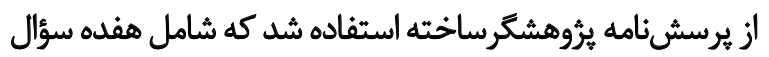


جدول r. نمره تبعيت از رثيم درمانى و مؤلفههاي آن در زنان مبتلا به ديابت باردارى (•\&ץ نفر)

\begin{tabular}{|c|c|}
\hline ميانكين土|نحر أفميار & مثغيرها \\
\hline$r \varphi / r \pm \Psi / N$ & ثمره تبعيت از رزيم غذايع \\
\hline$F / g \pm r / F$ & نمره هايش قند خون \\
\hline $1 / 8 \pm 1 / \Delta$ & نمره تبعيت از ورزش \\
\hline$r \cdot 1 \Delta \pm \Delta / \gamma$ & نمره كل تبعيت \\
\hline
\end{tabular}

خواندن و نوشتن نمره كل تبعيت از درمان بالاترى كسب كردند

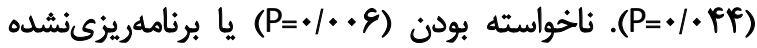

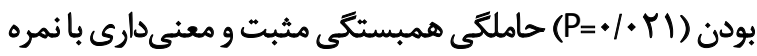

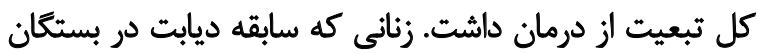

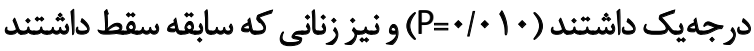

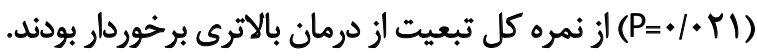

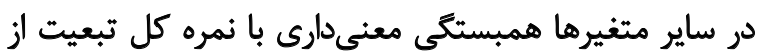
درمان ديده نشد (جدول شماره سم).

\section{$\Leftrightarrow$}

نتايج مطالعه حاضر نشان داد نمره تبعيت از رثيم غذاييى، نمره

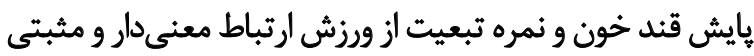

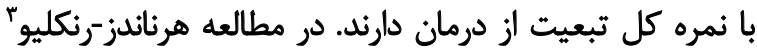

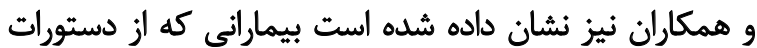

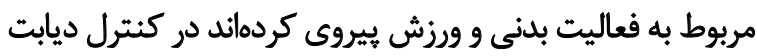

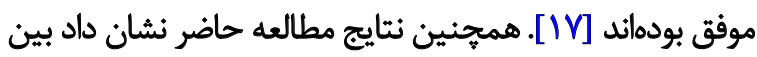

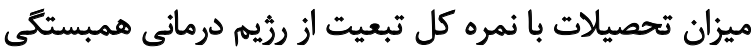

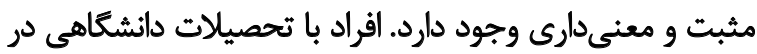

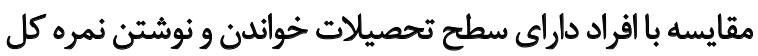
تبعيت از درمان بالاترى داشتند. در مطالعه عابدى و و همكاران

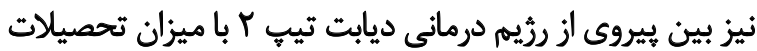

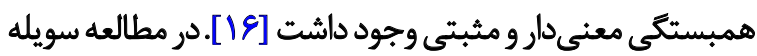

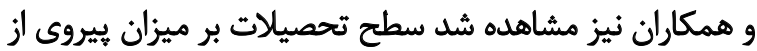

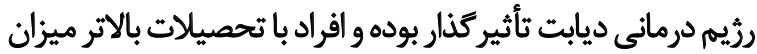

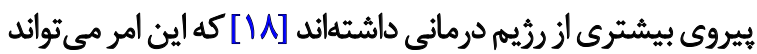

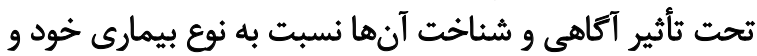

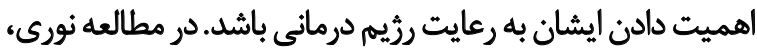

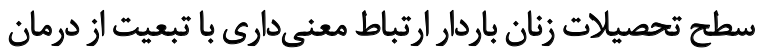

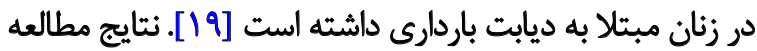

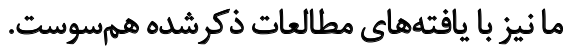
در مطالعه حاضر بين ميزان اطلاعات فرد (به كزارارش فرد) در

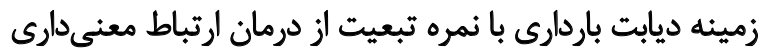

3. Hernández-Ronquillo
يرؤهشكرساخته تبعيت از درمان با شاخص روايى محتوايي'

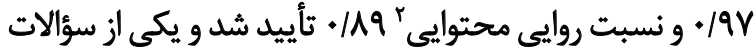

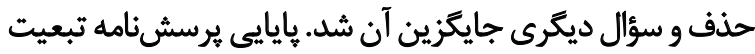

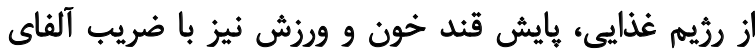

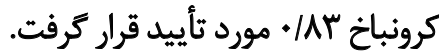

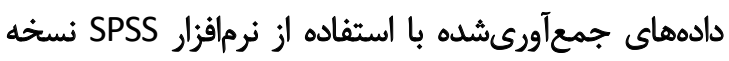

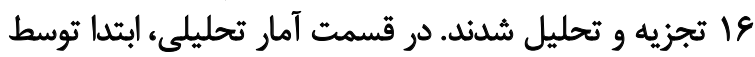

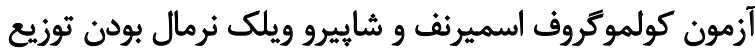

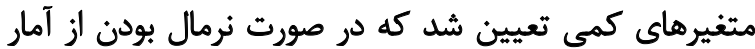

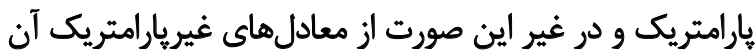

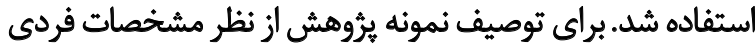

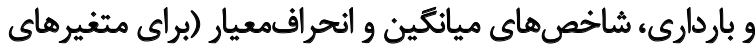

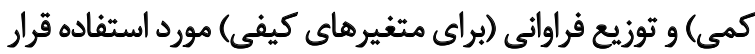

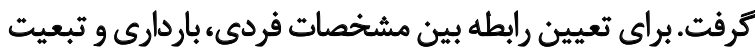

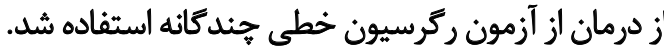

ياقتهها

توزيع فراوانى زنان مبتلابه ديابت باردارى مورد مطالعه بر حسب

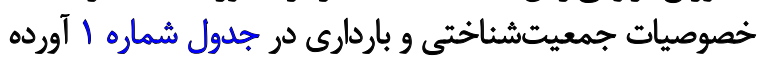
شيلهه أست.

ميانكين نمرات تبعيت از رزيم درمانى و مؤلفههاى آن در زنان مبتلا به ديابت باردارى در جدول شماره ب نشان داده شده است.

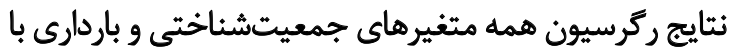

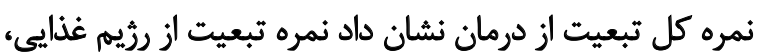

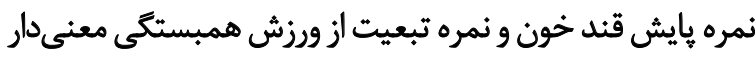

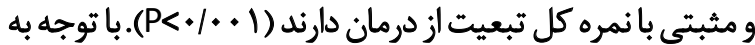

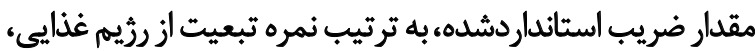

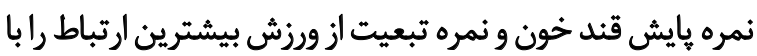

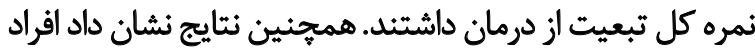

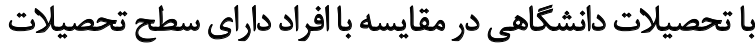

1. Content Validity Index (CVI)

2. Content Validity Ratio (CVR) 
جدول ب. نتايج تحليل ركرسيون خطى جندكَائه بررسى رابطه متغيرهاى جمعيتشناختى و مؤلفههاى تبعيت درمان با نمره كل تبعيت

\begin{tabular}{|c|c|c|c|c|c|}
\hline سطح معنىدارى & t آماره & استانداريبده & انحرافمعيار ضريب & ضريب & متغير هاى يُزوهش \\
\hline .1011 & $r / \Delta \Delta A$ & & $\cdot x+8$ & $1 / \times 49$ & مقدار ثابت \\
\hline$<. / .+1$ & A./rav & . & $\%$ & ./MT & نمره تبعيت از رثيم غذايى \\
\hline$<+1+\infty 1$ & $9 \cdot 19 \Delta 8$ & $.14+9$ & .1 .18 &.$/ Q 8$ & نمره هايش قند خون \\
\hline$<\cdot 1 \cdot .1$ & $r \Delta / / V^{m}$ &.$/ T \Delta 1$ & $.1 \cdot \pi$ &.$/ 98$ & نمره تبعيت از ورزش \\
\hline . late & $-/ 48$ &.$- / .+r$ & .10 .8 & $-* / .+r$ & سن \\
\hline.$/ r \cdot 1$ & - M/rAT & -.10 .9 & . NeF & $-* / K I$ & شعل (مرجع: خانهدار) \\
\hline$\cdot N+\Delta$ &.$/ m V A$ & $1 \% 0$ & - MEs & 1.84 & ابثلايي \\
\hline . /PAE & - 18q7 & $1 \% 1$ & - MEV & $.111 \mathrm{~V}$ & تحصيلات (مرجع: خواندن و نوشتن) راهنمايى و ديبرستان \\
\hline $.1+4$ & thert & 1.48 &.$/ r i r$ &.$/ 4 \pi$ & دانشكاهي \\
\hline.$/ M T$ & $-1 / 2 \pi y$ & $-4+4$ & $1+\mathrm{rag}$ & $-\pi / 4 \pi$ & درآمد ماهيانه (مرجع: كمثر از حد كفاف) در حلد كفاف \\
\hline$+/ N E$ & $-V / M T V$ & $-.1+.9$ &.$/ \mathrm{r}$ & $-* / m r$ & 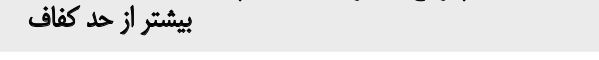 \\
\hline $\begin{array}{l}.1+14 \\
. / 411\end{array}$ & $\begin{array}{l}\text { IMAT } \\
\text {. ATrT }\end{array}$ & $\begin{array}{l}.1 \cdot 1 r \\
.1++\infty\end{array}$ & $\begin{array}{l}\cdot|+A| \\
\cdot \mid t+1\end{array}$ & $\begin{array}{l}.|N| F \mid \\
. / M T\end{array}$ & اطلاعات ديابت باردارى (مرجع: كم) \\
\hline $\begin{array}{l}.1 .09 \\
.1 .41\end{array}$ & $\begin{array}{l}\text { TMOP } \\
\text { YTMTI }\end{array}$ & $\begin{array}{l}\cdot 1 \cdot 11 \\
.1 \cdot 17\end{array}$ & $\begin{array}{l}. M \cdot V \\
. M E V\end{array}$ & $\begin{array}{l}. / \text { TY } \\
. / \text { MAV }\end{array}$ & ناخواسته \\
\hline .1819 & $\cdot / r \cdot$. & 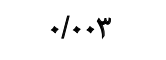 & $.1 .4 \cdot$ & $.1+14$ & ت ت تعلاد باردارى \\
\hline$+1+1$ & Y/DQV & $.1 \cdot 1 \mathrm{~V}$ & $\cdot 1 \cdot V^{n}$ &.$/ 19$ & سابقه ديابت در بستكان درجهيك (مرجع: خير) \\
\hline$. / \varphi+$. & $-* / A P r$ & $-.1 .+8$ & 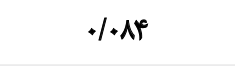 & $-* / * n$ & سابقه ديابت باردارى (مرجع: خير) \\
\hline. $\mid+r)$ & $-r / m T P$ & $-.1 \cdot 18$ & $1 \cdot 1 \Delta$ & $-* / 19$ & سابقه سقط (مرجع: خير) \\
\hline.$/ 1 \Delta \Delta 9$ &.$- / 1 \mathrm{~W}$ & $-* / * 1$ & / Mar &.$- / .+r$ & سابقه مردمزايع (مرجع: خير) \\
\hline ./qve & $1 . r$. &.+1 &.$/ 11 \%$ & . & سابقه نازايي (مرجع: خير) \\
\hline
\end{tabular}

معكوس و معني دارى ميان عدم انكيزه با تبعيت از رئيم داروييى

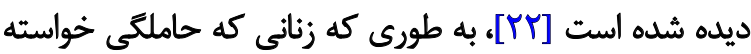

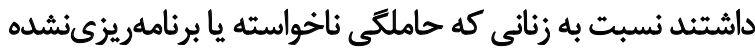

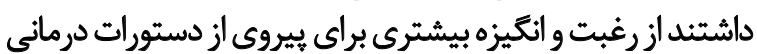
برخوردار بودند.

زناني كه سابقه ديابت در بستئان درجهيك داشتيند، از نمره

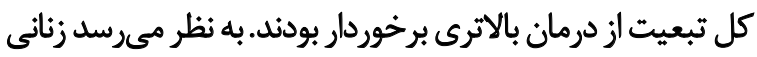

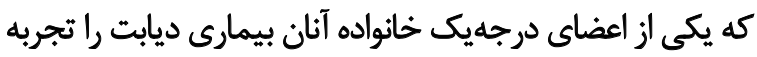

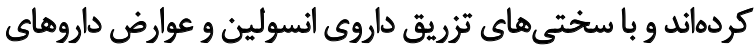

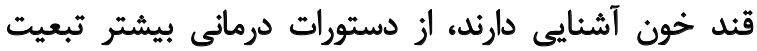

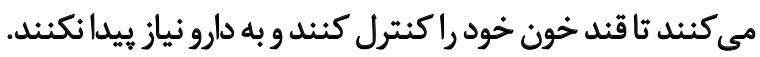

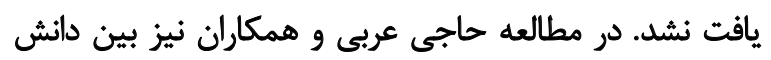

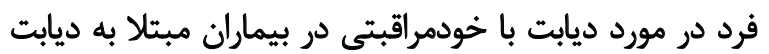

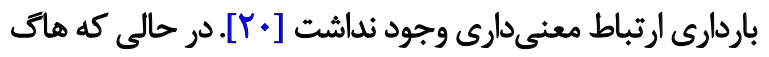

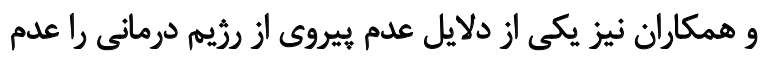

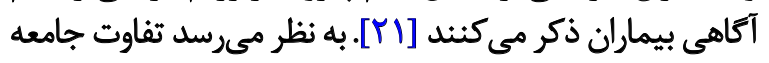
يروهش از نظر فرهنكى در اين عدم همسو بودن نقش دان داشتي

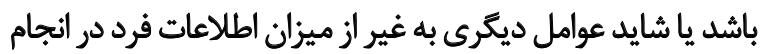

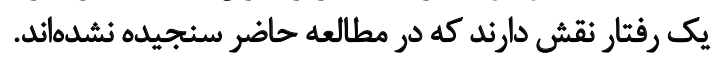

در مطالعه حاضر ناخواسته بودن يا برنامهريزىنشده بودن

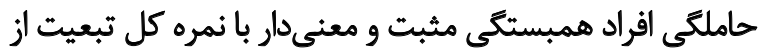

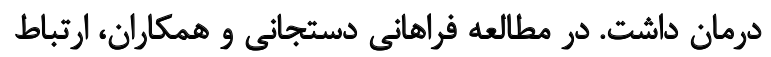




$$
\text { the who }
$$

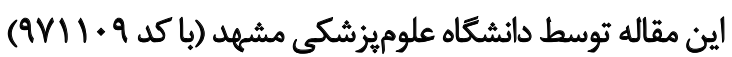

$$
\text { حمايت مالى شده است. }
$$

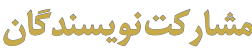

مفهومسازى: مريم مرادى، زهرا عابديان؛ روش : يروهش و و

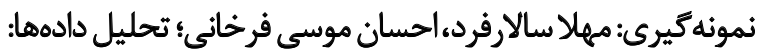

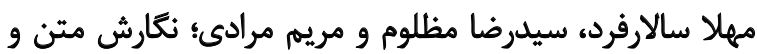

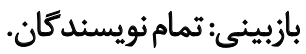

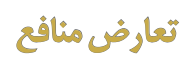

نويسندًان تصريح مى كنند هيجگونه تعارضى در منافع وجود

$$
\text { 落 }
$$

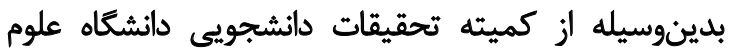

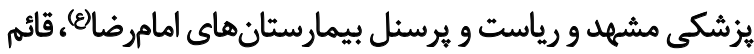

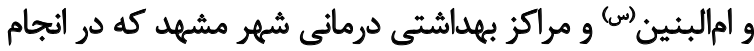
اين يثوهش ما را يارى كردند، نهايت تشكر را دارينم.
در مطالعه آريان سوئيتينك و همكاران، بين سابقه ديابت باردارى

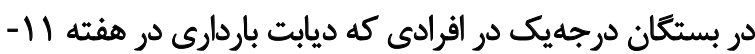

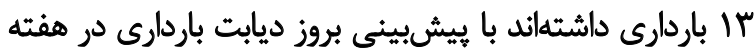

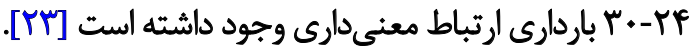

در مطالعه حاضر زنانى كه سابقه سقط داشتند، از نمره كل

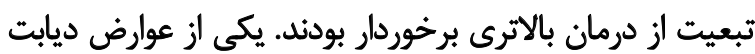

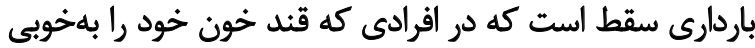

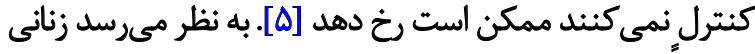

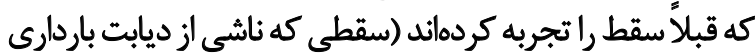

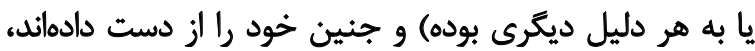

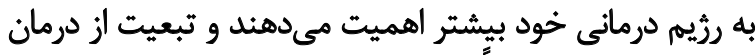

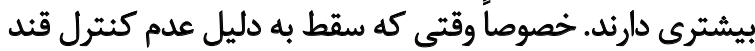

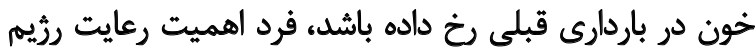

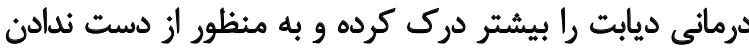
جنين خود تبعيت از درمان بيشترى دارد درد نتايج مطالعه حاضر نشان داد برخى از مشخصات

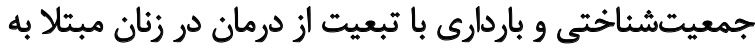

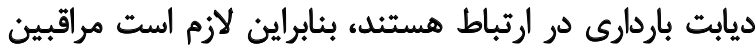

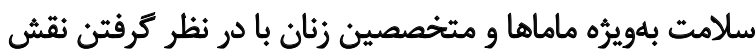
اين عوامل باعث بهبود تبعيت از درمان در بيماران شوندان باندان

$$
\text { نتيجليكيرى }
$$

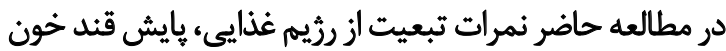

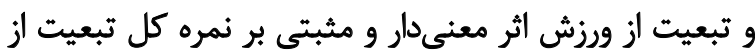

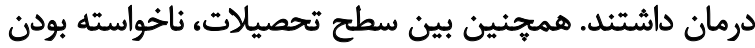

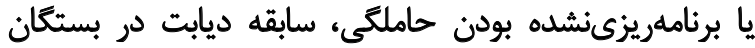

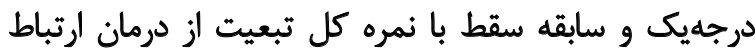

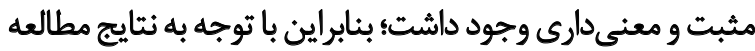

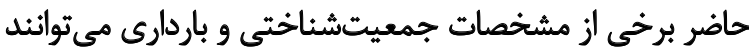

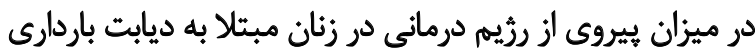

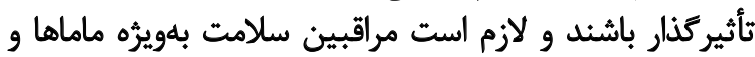

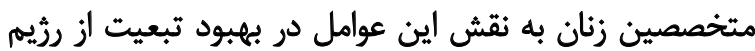

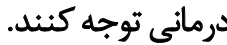

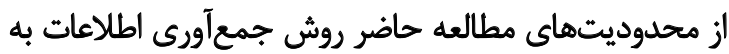

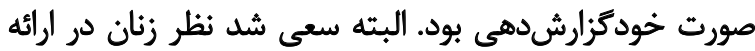
باسخهاى درست و واقعى جلب شود.

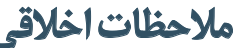

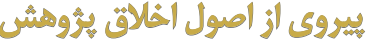

IR.MUMS.NURSE.REC.1397.091 اين مطالعه باكداخلاق در كميته اخلاق دانشعاه علوميزشكى مشهد به ثبت رسيد. 


\section{References}

[1] Cunningham FG, Leveno KJ, Bloom SL, Dashe JS, Hoffman BL, Casey BM, et al. Williams Obstetrics. New York City: McGraw-Hill Education; 2018. https://books.google.com/books?id=mhOdAQAACAAJ\&dq=Willia ms+Obstetrics, +25 e \&hl=en\&sa=X\&ved=2ahUKEwjJ-_vx5unxAhUBCxoKHVZjDCoQ6AEwAHoECAsQAg

[2] Jamilian M, Afshar R. [Effects of combined evening primrose oil and vitamin $D$ intake on hs-CRP, oxidative stress and pregnancy outcomes in women with gestational diabetes (Persian)]. J Arak Univ Med Sci. 2017; 19(12):43-51. http://jams.arakmu.ac.ir/article-1-4736-en.html

[3] Hunt KJ, Schuller KL. The increasing prevalence of diabetes in pregnancy. Obstet Gynecol Clin North Am. 2007; 34.(2):173-99. [DOI:10.1016/j. ogc.2007.03.002.] [PMID] [PMCID]

[4] Jafari-Shobeiri M, Ghojazadeh M, Azami-Aghdash S, Naghavi-Behzad M, Piri R, Pourali-Akbar Y, Nasrollah-Zadeh R, et al. Prevalence and risk factors of gestational diabetes in Iran: A systematic review and metaanalysis. Iran J Public Health. 2015; 44(8):1036-44. [PMID] [PMCID]

[5] Salzer L, Yogev Y. Complications of gestational diabetes. In: Petry CJ, editor. Gestational diabetes: Origins, complications, and treatment. Florida: CRC Press; 2014. p.95. [DOI:10.1201/b16474-9]

[6] Kooshyar H, Shoorvazi M, Dalir Z, Hosseini M. [Health literacy and its relationship with medical adherence and health-related quality of life in diabetic community-residing elderly (Persian)]. J Mazandaran Univ Med Sci. 2014; 23(1):134-43. http://jmums.mazums.ac.ir/article1-3439-en.html

[7] Osterberg L, Blaschke T. Adherence to medication. N Engl J Med. 2005; 353(5):487-97.[DOI:10.1056/NEJMra050100] [PMID]

[8] Shrivastava SR, Shrivastava PS, Ramasamy J. Role of self-care in management of diabetes mellitus. J Diabetes Metab Disord. 2013; 12(1):14. [DOI:10.1186/2251-6581-12-14.] [PMID] [PMCID]

[9] Rezaie Kargar F, Karbandi S, Hassanabadi H, Esmaili H. [Type of Personality and the Amount of Adherence to Recommended Regimens in Diabetic Patients (Persian)]. Psychiatry Clin Psychol Iran. 2006; 11(4):441-8. http://ijpcp.iums.ac.ir/article-1-50-fa.html

[10] Karimi S, Mansouri P, shahdadi H, Pakizedel P. [The effect of health belief model-based education on adherence to the dietary regimen in pregnant women with gestational diabetes (Persian)]. J Diabetes Nurs. 2016; 4(4):1-9. http://jdn.zbmu.ac.ir/article-1-216-fa.html

[11] Rouhi Balasi I, Paryad E, Kazemnezhad Leili E, Booraki SH, SadeghiMeibodi A, Nassiri Sheikhani N. [Study status of care adherence and its related factors in patients undergoing (Persian)]. J Holist Nurs Midwifery. 2015; 25(3):34-45. http://hnmj.gums.ac.ir/article-1-525-en.html

[12] Bosworth HB, Oddone EZ, Weinberger M. Patient treatment adherence: Concepts, interventions, and measurement. New York: Psychology Press; 2006. [DOI:10.4324/9781410615626]

[13] Lerman I. Adherence to treatment: The key for avoiding long term complications of diabetes. Arch Med Res. 2005; 36(3):300-6. [DOI:10.1016/j.arcmed.2004.12.001.] [PMID]

[14] Hashemi M, haghighat S, bagheri M, Akbari M, Niknejad R.[Factors affecting adherence to the treatment in patients under hemodialysis: A qualitative content analysis (Persian)]. Nurs Midwifery J. 2020; 18(2):141-56. http://unmf.umsu.ac.ir/article-1-3899-en.htm

[15] Browne T, Merighi JR. Barriers to adult hemodialysis patients' selfmanagement of oral medications. Am J Kidney Dis. 2010; 56(3):547-57. [DOI:10.1053/j.ajkd.2010.03.002.] [PMID]
[16] Abedi H, Rezayi A, Alimohammadi N, GhodusiA, Maghroun A. [An investigation of demographic and social factors affecting compliance with therapeutic regimens in type 2 diabetic patients (Persian)]. J Diabetes Nurs. 2015; 3(2):66-81. http://jdn.zbmu.ac.ir/article-1-116-fa.htm

[17] Hernández-Ronquillo L, Téllez-Zenteno JF, Garduño-Espinosa J, GonzálezAcevez E. Factors associated with therapy noncompliance in type-2 diabetes patients. Salud Publica Mex. 2003; 45(3):191-7. [DOI:10.1590/ S0036-36342003000300008] [PMID]

[18] Sweileh WM, Aker O, Hamooz S. Rate of compliance among patients with diabetes mellitus and hypertension. An-Najah Univ J Res. 2005; 19(1). https://repository.najah.edu/bitstream/handle/20.500.11888/1860/rate-compliance-among-patients-diabetesmellitus-and-hypertension.pdf?sequence=1\&isAllowed=y

[19] Nouri A. [Investigating the socio-economic factors affecting the compliance of patients with gestational diabetes with the doctor's instructions in the clients of Kerman Diabetes Center in 2013 (Persian)] [MS. thesis]. Kerman: Kerman University of Medical Sciences; 2015. http:// eprints.kmu.ac.ir/id/eprint/21727

[20] Haji-Arabi E, Nobahar M, Ghorbani R. [Relationship between depression and knowledge about diabetes with the amount of self-care in patients with type 2 diabetes (Persian)]. Koomesh. 2018; 20(2):210-20. http://koomeshjournal.semums.ac.ir/article-1-4164-fa.html.

[21] Haque M, Emerson SH, Dennison CR, Navsa M, Levitt NS. Barriers to initiating insulin therapy in patients with type 2 diabetes mellitus in public-sector primary health care centres in Cape Town. S Afr Med J. 2005; 95(10):798-802. [PMID]

[22] Farahani Dastjani F, Shamsi M, Khorsandi M, Rezanfar RMZ. [Investigating the relationship between the barriers and non-compliance with the drug regimen in type 2 diabetic patients in Arak in 2014 (Persian)] Daneshvar Med. 2015; 23(2):49-58. https://www.sid.ir/fa/journal/ ViewPaper.aspx?id=246071

[23] Sweeting AN, Appelblom H, Ross GP, Wong J, Kouru H, Williams PF, et al. First trimester prediction of gestational diabetes mellitus: A clinical model based on maternal demographic parameters. Diabetes Res Clin Pract. 2017; 127:44-50. [DOI:10.1016/j.diabres.2017.02.036] [PMID] 